\title{
Expansively Hemorrhagic Foreign Body Granuloma at the Pelvis Caused by Microscopic Materials
}

\author{
Masatoshi Morimoto ${ }^{1}$, Mitsuhiko Takahashi ${ }^{1 *}$, Nori Sato ${ }^{1}$, Toshihiko Nishisho ${ }^{1}$, Seiko Kagawa ${ }^{2}$, \\ Eiji Kudo $^{2}$, Shoichiro Takao ${ }^{3}$, Natsuo Yasui ${ }^{1}$ \\ ${ }^{1}$ Department of Orthopaedic Surgery, Institute of Health Biosciences, University of Tokushima Graduate School, Tokushima, Japan; \\ ${ }^{2}$ Department of Human Pathology, Institute of Health Biosciences, University of Tokushima Graduate School, Tokushima, Japan; \\ ${ }^{3}$ Department of Radiology, Institute of Health Biosciences, University of Tokushima Graduate School, Tokushima, Japan. \\ Email: *w-tk@umin.ac.jp
}

Received December $14^{\text {th }}, 2011$; revised January $24^{\text {th }}, 2012$; accepted February $9^{\text {th }}, 2012$

\begin{abstract}
A foreign body granuloma is a mass lesion consisting of a foreign body with host reaction. Its diagnosis is not always easy in respect of its similarity to soft tissue sarcoma. The most common foreign body is surgical sponge which can be detectable macroscopically. We present a foreign body granuloma in the left buttock caused by microscopic surgical materials that developed into a large lesion. An 80-year-old man with a history of surgery for dislocation of the left hip 20 years earlier, presented with a slowly growing mass that had expanded rapidly over the preceding 2 months. Histological examination revealed organizing granulation tissue surrounding the scattered foreign materials. One should be aware of foreign body granuloma at previously operated sites even without a retained macroscopic gauze sponge.
\end{abstract}

Keywords: Expanding Hematoma; Foreign Body Granuloma; Histologic Examination; Magnetic Resonance Imaging

\section{Introduction}

A foreign body granuloma (also called gossypiboma) is a mass lesion consisting of a foreign body with host reaction to the foreign body [1]. The most common cause of foreign body granuloma is surgical sponge implanted during surgical intervention. It is less common after orthopedic surgery compared with abdominal surgery. Clinical symptoms often emerge many years after the surgery. Foreign body granuloma is difficult to distinguish from soft tissue sarcomas, which are malignant tumor [13]. Rapid growth of the lesion is one of the signs for malignancy. We present the case of patient with foreign body granuloma in the left buttock, which had expanded rapidly. Surprisingly, microscopic, but not macroscopic, foreign bodies were detected histologically, which have not been described in the previous literature.

\section{Case Report}

An 80-year-old man presented at our institute with a soft tissue tumor in the left buttock which was noticed 2 years earlier and expanded rapidly over the recent 2 months. He was involved in a traffic accident 20 years earlier (at 60 years of age) resulting in a dislocation of the left hip joint with an ipsilateral acetabular fracture. The disloca-

"Corresponding author. tion was reduced surgically at a local orthopaedic hospital, while the acetabular fracture was treated non-surgically after the hip reduction. He had been suffering from left hip pain since that accident and been informed as having secondary osteoarthritis of the hip. The suture material used for deep tissue at the hospital was identified as a non-absorbable braided nylon (Surgilon ${ }^{\circledR}$ ). Additional materials used at the time of surgery were unknown.

On physical examination, the skin over the tumor was tense with varicoses and showed cyanosis. Motion of the left hip was restricted in all motion planes. Laboratory examinations showed unremarkable findings with a normal cell count and normal biochemistry.

A plain X-ray revealed advanced osteoarthritis of the left hip with proximal migration of the femoral head and an expanding soft tissue contour outside of the ipsilateral ilium (Figure 1(A)). Computed tomography (CT) with contrast enhancement demonstrated an irregularly enhanced lesion in the region of the left gluteus muscles (Figure 1(B)). CT also revealed advanced osteoarthritis of the left hip associated with retained posterior subluxation of the hip. Magnetic resonance (MR) imaging showed an inhomogeneous lesion measuring $14 \times 11 \times 8 \mathrm{~cm}$, which was excluding the gluteus muscles (Figures 2(A)(C)). The lesion contacted the ilium, the hip joint capsule 


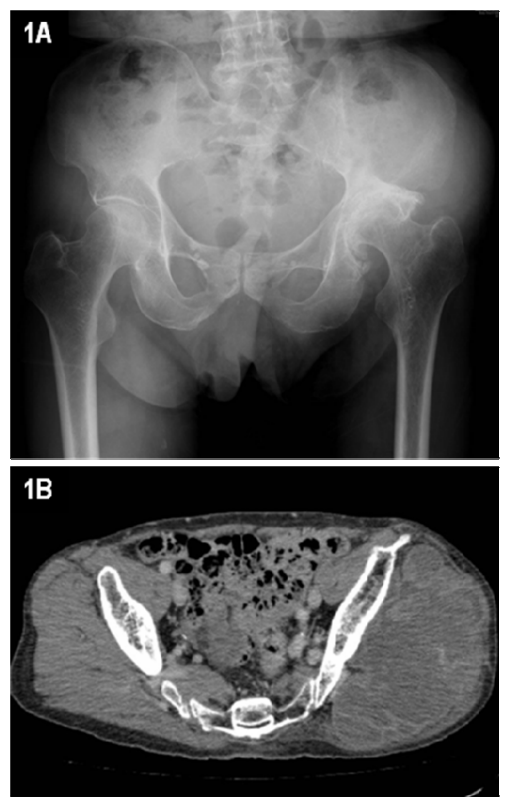

Figure 1. A radiograph indicating advanced osteoarthritis of the left hip and an expanding soft tissue contour on the ipsilateral ilium (A). Computed tomography (CT) images at the ala of ilium (B).

and the greater trochanter. The lesion had a hypointense rim with a central hyperintense zone on T1-weighted images, and a heterogenous signal from high to low intensity with several fluid-fluid levels on T2-weighted images. Gadolinium-enhanced images of the lesion showed peripheral enhancement. MR intensity change of bone marrow was partly seen in the ala of ilium but never in the femoral head. A high grade sarcoma with intralesional hematoma was suspected from these findings. Considering the tense skin over the tumor predisposing skin rupture, as well as the advanced age of the patient with critical dilated cardiomyopathy, we planned marginal excision without preceding histological examination to alleviate his symptoms.

Transcatheter arterial embolization was performed to prevent massive intra-operative bleeding 2 days before the planned excision. The lesion was peripherally hypervascular with extravasations from the superior and inferior gluteal arteries (Figure 2(D)). Tumor stain disappeared after the embolization. The lesion was located deep within the thinned gluteus muscle and was in contact with the ilium and the joint capsule. Nevertheless, it was entirely encapsulated and able to be marginally resected. No perforation or tear was found on the hip joint capsule and periosteum of the ilium. The resected specimen was a well-defined and slightly firm mass measuring $16 \times 10 \times 7 \mathrm{~cm}$ (Figure 3(A)). It consisted of paste- like previous hemorrhage and solid components (fibrous walls and septums) that was friable (Figure 3(B)). Any apparent foreign materials were detected at this time point.
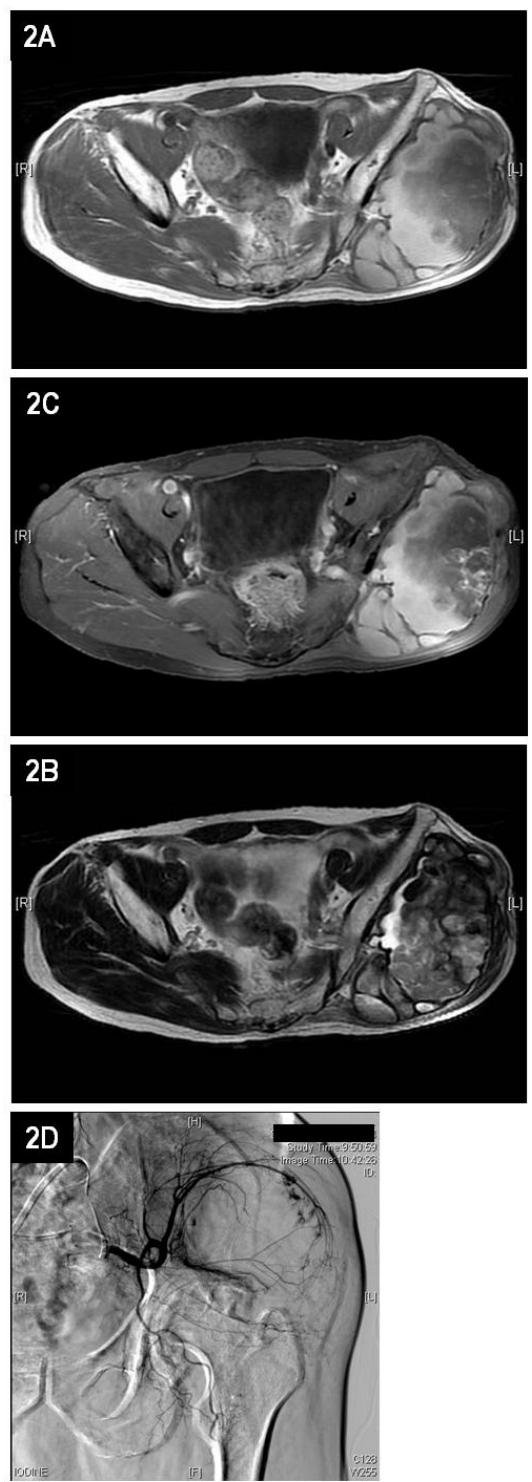

Figure 2. Axial views of preoperative magnetic resonance (MR) images; T1-weighted image (A), T2-weighted image (B) and gadolinium-enhanced T1-weighted image with fatsaturation (C). Selective angiography of the left internal iliac artery (D).

Histologic examination of these solid components showed organizing granuloma cells surrounding scattered foreign bodies which showed a white birefringence under polarized light (Figures 4(A) and (B)). In high-power field, multinucleated giant cells phagocytized these foreign bodies. The granuloma cells including the giant cells had strong immunostaining for CD68 but not for S-100 and contained PAS-positive granules in their cytosols (not shown). The lesion also contained fibrous elements and abundant blood vessels with various sizes. No amyloid deposition in the blood vessels was proved by applegreen birefringence on Congo red staining, despite the presence of amyloid deposition between fibrous matrix 

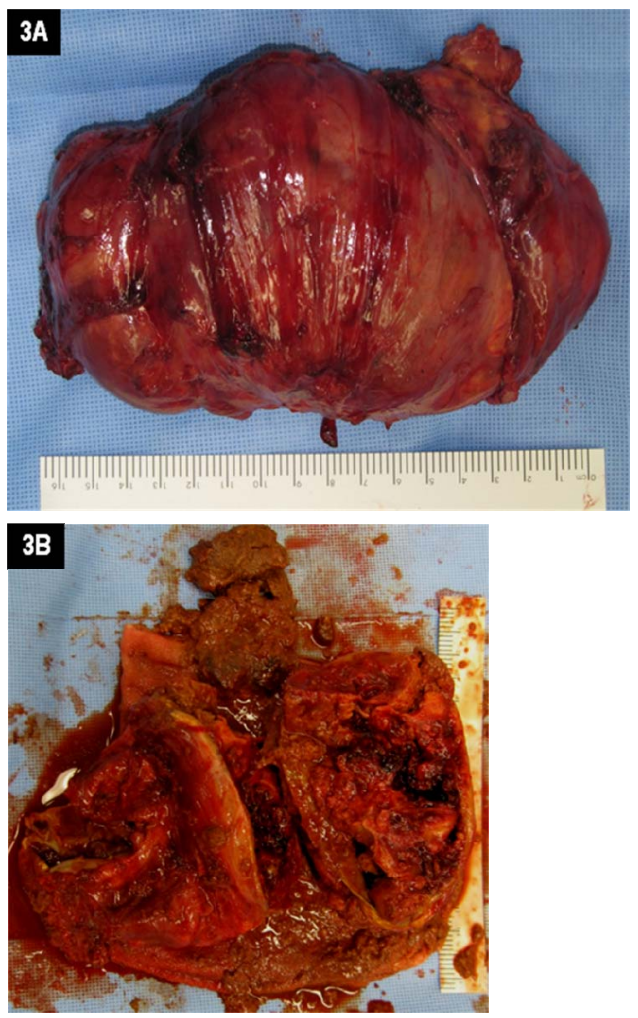

Figure 3. Macroscopic appearance of the resected tumor (A) and its contents (B).

matrix elements (not shown). There were otherwise necroses probably due to preoperative embolization and no signs of malignancy in the resected specimen. We diagnosed the lesion as a foreign body granuloma resulting from non-absorbable materials used 20 years previously and modified by intralesional expanding hematomas. At one year after surgery, the patient was free from recurrence despite the presence of hip pain probably due to the remaining osteoarthritis.

\section{Discussion}

Foreign body granuloma arises in response to surgically implanted foreign materials [1]. Wilson et al. first described a case of a retained foreign body after laparotomy [4]. Since then, foreign body granulomas have been recognized as a relatively common complication after surgical procedures such as splenectomy, gastrectomy, colectomy, herniorrhaphy, prostatectomy, cholecystectomy, brain surgery, and bladder surgery [5]. For the incidence of foreign body granuloma, abdominal surgery accounts for $52 \%$ of all the cases, gynecologic for $22 \%$ and urologic and vascular for $10 \%$ [6]. However, it is less common after orthopedic surgery, accounting for only $6 \%$ of reported cases.

In the present case, we were able to identify microscopic, but not macroscopic, foreign bodies. Gauze sponge
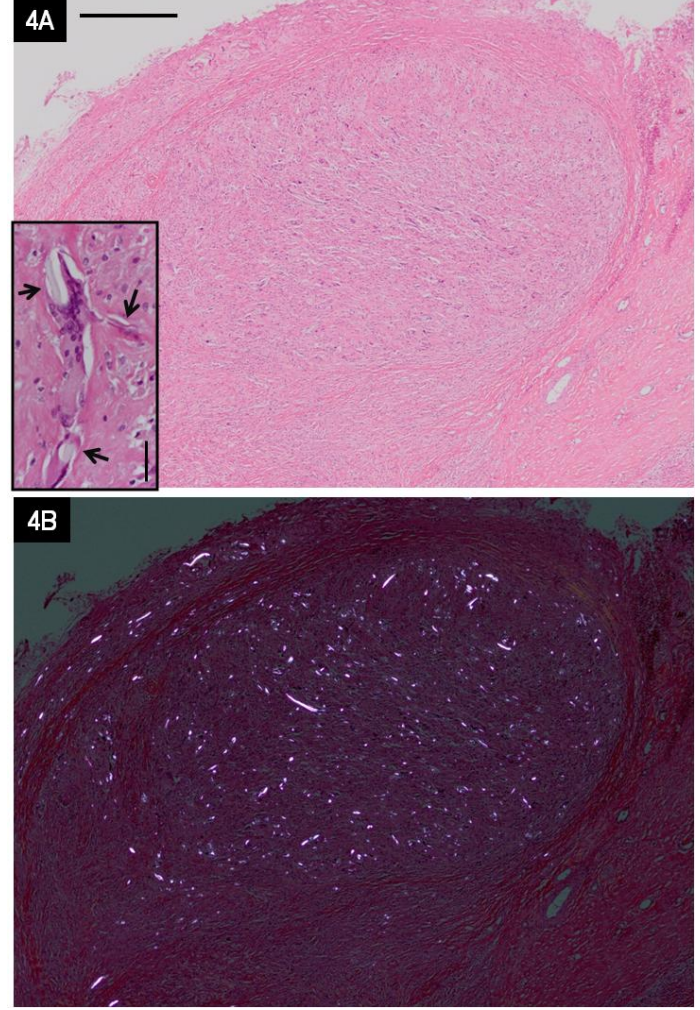

Figure 4. Microscopic examination of the lesion revealed granulation tissue containing the scattered suture materials, which was surrounded by fibrous elements (the scale bar represents $500 \mu \mathrm{m}$, (A) Inset showed presence of giant cells, which phagocytosed the suture material (arrows, the scale bar represents $50 \mu \mathrm{m}$ ). The same section as (A) under polarized light (B).

has been reported as the prime cause of foreign body granuloma. There were microscopic foreign materials scattered in the lesion, which were phagocytosed by host cells. Although macrophages can phagocytose foreign bodies [7], non-absorbable surgical materials might be retained for a long time in the body because of inability to degrade these materials $[8,9]$. We were unable to identify the substance directly due to scattered and tiny samples obtained. Comparison of birefringence with representative surgical fibril materials under polarized light microscopy demonstrated that the substance from the lesion had similar pattern to non-absorbable braided nylon and cotton (gaze sponge) but not silk or polyester (Ethibond $^{\circledR}$ ) sutures. The cause of foreign body granuloma of more than $10 \mathrm{~cm}$ in diameter is a macroscopically-identified gauze sponge only. To the best of our knowledge, the present case is the first in which a foreign body granuloma of larger than $10 \mathrm{~cm}$ in diameter was caused by foreign materials detected only microscopically, although there was a possibility that gaze sponge could have been dismantled into tiny fragments by the time of surgical resection. 
Nevertheless, intralesional hematoma contributed to the expansion of lesion in this case. Expanding hematoma can occur in various conditions including tumorous lesions and often complicates the etiology of the original lesion. The reason why hemorrhage occurred after a nearly 20-year asymptomatic course is unknown. One explanation was the hypervascularity detected on the histological sections, which agreed with the previous report [10]. There was no vascular amyloid deposition, which was found to lead to hemorrhage [11]. The encapsulated lesion would deny the theory that hematoma resulted from the surrounding tissue such as hip joint. Furthermore, the patient had never been given any anticoagulant agents such as aspirin or warfarin despite having dilated cardiomyopathy.

Several authors have reported MR imaging findings of foreign body granuloma. Kominami et al. described wavy low-signal-intensity lines [10]. Sakayama et al. reported a folded fabric structure and whirl-like appearance [2]. However, these findings are applicable to those caused by macroscopic surgical sponge. The foreign body granulomas reported in the orthopedic field were generally difficult to differentiate from soft tissue sarcomas [1-3]. Despite awareness of intralesional hematoma, we preoperatively diagnosed the lesion as a malignant tumor on the basis of the following MR findings: 1) tumor of over $10 \mathrm{~cm}$ in maximum diameter with deep location in soft tissue; 2) absence of a folded fabric structure and whirllike appearance; 3) heterogeneous intensity on T2-weighted images; 4) osteolytic erosion of the left iliac bone; and 5) irregular enhancement of the lesion after gadolinium injection.

For the treatment, the large lesion at risk of local skin rupture prevented us from choosing less invasive plans such as irradiation. Due to the advanced age of patient with critical complication in addition to the local skin condition, we did not perform preceding histological examination via biopsy. We emphasize, in agreement with others, that this is not the case in most patients with similar lesions; that is, histological examination is necessary to determine the surgical plan [12,13]. It is difficult to diagnose as a foreign body granuloma by imaging techniques alone [12]. On the other hand, undifferentiated pleomorphic sarcoma and angiosarcoma have been reported to develop occasionally from a foreign body granuloma [14,15]. Thus, proper surgical planning including prior histological examination is necessary. One should be aware of foreign body granuloma at previously operated sites even without a retained macroscopic gauze sponge and with rapid growth after years of asymptomatic course.

\section{REFERENCES}

[1] B. Mboti, M. Gebhart, D. Larsimont and K. Abdelkafi,
“Textiloma of the Thigh Presenting as a Sarcoma," Acta Orthopaedica Belgica, Vol. 67, No. 5, 2001, pp. 513-518.

[2] K. Sakayama, T. Fujibuchi, Y. Sugawara, et al., “A 40Year-Old Gossypiboma (Foreign Body Granuloma) Mimicking a Malignant Femoral Surface tumor,” Skeletal Radiology, Vol. 34, No. 4, 2005, pp. 221-224. doi:10.1007/s00256-004-0821-7

[3] D. F. Kalbermatten, N. T. Kalbermatten and R. Hertel, "Cotton-Induced Pseudotumor of the Femur," Skeletal Radiology, Vol. 30, No. 7, 2001, pp. 415-417. doi:10.1007/s002560100358

[4] C. Wilson, "Foreign Bodies Left in the Abdomen after Laparotomy," Gynecologic Treatment, Vol. 9, 1884, pp. 109-112.

[5] G. Fink, P. Herskovitz, M. Nili, et al., "Suture Granuloma Simulating Lung Neoplasm Occurring after Segmentectomy,” Thorax, Vol. 48, No. 4, 1993, pp. 405-406. doi:10.1136/thx.48.4.405

[6] E. Mouhsine, R. Garofalo, A. Cikes and P. F. Leyvraz, "Leg Textiloma. A Case Report," Medical Principles and Practice, Vol. 15, No. 4, 2006, pp. 312-315. doi:10.1159/000092998

[7] Y. Tsuchida and T. Oyamada, "Experimental Pathological Study on the Postoperative Suture Granuloma in Rats," Journal of the Japan Veterinary Medical Association, Vol. 61, No. 11, 2008, pp. 873-839.

[8] S. R. Kim, H. K. Baik and Y. W. Park, "Retained Surgical Sponge Presenting as a Pelvic Tumor after 25 Years," International Journal of Gynecology \& Obstetrics, Vol. 82, No. 2, 2003, pp. 223-225. doi:10.1016/S0020-7292(03)00139-5

[9] R. Kobayashi, T. Fukui, A. Tatematsu, Y. Yatabe and T. Mitsudomi, "A Suture Granuloma of the Lung That Occurred 19 Years after Surgery for Mucoepidermoid Tumor," Journal of the Japanese Association for Chest Surgery, Vol. 23, No. 2, 2009, pp. 165-168. doi:10.2995/jacsurg.23.165

[10] M. Kominami, A. Fujikawa, T. Tamura, Y. Naoi and O. Horikawa, "Retained Surgical Sponge in the Thigh: Report of the Third Known case in the Limb," Radiation Medicine, Vol. 21, No. 5, 2003, pp. 220-222.

[11] M. O. McCarron and J. A. Nicoll, "Cerebral Amyloid Angiopathy and Thrombolysis-Related Intracerebral Haemorrhage,” Lancet Neurology, Vol. 3, No. 8, 2004, pp. 484 492. doi:10.1016/S1474-4422(04)00825-7

[12] L. Kopka, U. Fischer, A. J. Gross, et al., "CT of Retained Surgical Sponges (Textilomas): Pitfalls in Detection and Evaluation,” Journal of Computer Assisted Tomography, Vol. 20, No. 6, 1996, pp. 919-923. doi:10.1097/00004728-199611000-00009

[13] A. I. Okten, M. Adam and Y. Gezercan, "Textiloma: A Case of Foreign Body Mimicking a Spinal Mass,” European Spine Journal, Vol. 15, No. S5, 2006, pp. m626-629. doi:10.1007/s00586-006-0136-6

[14] T. Nishida, N. Nishiyama, Y. Kawata, et al., "Mediastinal Malignant Fibrous Histiocytoma Developing from a Foreign Body Granuloma,” Japanese Journal of Thoracic and Cardiovascular Surgery, Vol. 53, No. 10, 2005, pp. 583- 
586. doi:10.1007/s11748-005-0074-y

[15] T. A. Jennings, L. Peterson, C. A. Axiotis, et al., “Angiosarcoma Associated with Foreign Body Material. A Report of Three Cases,” Cancer, Vol. 62, No. 11, 1988, pp.
2436-2444.

doi:10.1002/1097-0142(19881201)62:11<2436::AID-CN CR2820621132>3.0.CO;2-J 